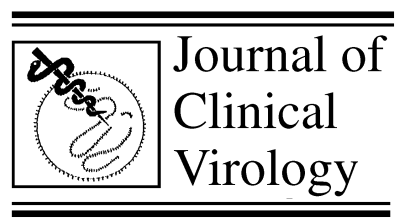

\title{
Baculovirus expression of parvovirus B19 (B19V) NS1: utility in confirming recent infection
}

\author{
O. Ennis, A. Corcoran, K. Kavanagh, B.P. Mahon, S. Doyle* \\ Department of Biology, National University of Ireland, Maynooth, Co. Kildare, Ireland
}

Received 10 July 2000; received in revised form 26 February 2001; accepted 8 March 2001

\begin{abstract}
Background: The presence of anti-parvovirus B19 (B19V) IgM against viral capsid proteins (VP1 and VP2) has long been used to detect recent infection. The utility of antibodies directed against B19V NS1 protein has received less attention as a serological indicator of recent infection, although anti-B19V NS1 IgG has been associated with persistent infection. Objectives: To elucidate the role of anti-B19V NS1 antibody detection in recent infection, full-length B19V NS1 was expressed and purified. The resultant antigen was used to develop both Western blot assays and microplate ELISA for the detection of NS1 antibodies. Study design: Serum specimens were obtained from individuals recently infected with B19V (children $(n=16)$, adults $(n=40))$ and from 17 individuals with no evidence of recent B19V infection. All specimens were screened for anti-B19V NS1 IgG and IgM. Results: It was observed that $68.8 \%$ (11/16) of children recently infected with B19V were anti-B19V NS1 IgG seropositive. Furthermore, 27.5\% $(11 / 40)$ anti-B19V VP2 IgM positive specimens also contained anti-B19V NS1 IgM when tested by ELISA, while no reactivity was observed following Western blot analysis, possibly due to the absence of conformational epitopes. Conclusions: Anti-B19V NS1 IgM detection may have utility in the confirmation of recent infection with B19V. (C) 2001 Elsevier Science B.V. All rights reserved.
\end{abstract}

Keywords: Baculovirus; ELISA; Epitopes; Immunocompromised; Pregnancy

\section{Introduction}

Since its discovery in 1975, parvovirus B19 (B19V) has been identified as the causative agent of a number of clinical conditions in humans (Anderson et al., 1985; Kurtzman et al., 1987). The spectrum of symptoms caused by B19V, in-

\footnotetext{
* Corresponding author. Tel.: + 353-1-7083858; fax: + 3531-7083845.

E-mail address: sean.doyle@may.ie (S. Doyle).
}

cluding rash, arthralgia and transient aplastic crisis, are generally self-limiting in healthy individuals. However, serious complications due to viral infection may arise in certain populations including pregnant women, immunocompromised patients and individuals suffering from sickle cell disease or other blood-related disorders (Jordan, 1996; Török, 1997).

The B19V capsid is composed of two structural proteins, VP1 $(83 \mathrm{kDa})$ and VP2 $(53 \mathrm{kDa})$, with VP2 comprising approximately $95 \%$ of total cap- 
sid protein (Ozawa and Young, 1987). Both of these antigens have been expressed in eukaryotic expression systems in order to assess their potential use as diagnostic reagents for B19V infection (Brown et al., 1990). This work has culminated in the recent demonstration of the absolute requirement for intact capsid structure for optimal detection of anti-B19V VP2 IgG (Kerr et al., 1999).

B19V NS1 had received much less attention as a marker of $\mathrm{B} 19 \mathrm{~V}$ infection until the proposal that anti-B19V NS1 IgG may be a marker of persistent infection (von Poblotzki et al., 1995a,b). Recent studies have demonstrated a range of $21.7-36 \%$ NS1 IgG seropositivity in control subjects previously infected with B19V (Venturoli et al., 1998; Jones et al., 1999; Hemauer et al., 2000). An anti-B19V NS1 IgG seropositivity of $12.5 \%(5 / 40)$ has been observed in recently infected individuals, and a similarly low rate of anti-B19V NS1 IgG positivity has been reported in acutely infected immunocompetent individuals $(5 / 43 ; 11 \%)$ (Jones et al., 1999; Hemauer et al., 2000). Most of these studies on the immune response to B19V NS1 have focused on the diagnostic utility of anti-B19V NS1 IgG as a marker of specific B19V-related disease or persistent infection, whereby the level of anti-B19V NS1 IgG positivity may be as high as $61 \%(24 / 39)$ and $80 \%(4 / 5)$, respectively, in cases of B19V infection during pregnancy or chronic B19V infection in immunocompetent individuals (Hemauer et al., 2000). Interestingly, all of these studies have been performed using E. coli expressed NS1 fragments as the detection antigen. Furthermore, no evidence of anti-B19V NS1 IgM has been found from specimens taken during recent B19V infection (Hemauer et al., 2000). A limited evaluation of baculovirus-expressed NS1 for B19V serological studies has been reported, whereby $12 \%(3 / 25)$ of specimens obtained from arthropathy patients were antiB19V NS1 IgG positive by Western blot analysis (Hicks et al., 1996). These authors also noted that an anti-B19V IgG positive serum pool tested negative for anti-B19V NS1 IgM, as expected. In this study, we sought to determine the utility of the full-length B19V NS1 protein produced in the baculovirus expression system for the determination of recent infection with $\mathrm{B} 19 \mathrm{~V}$ via the analysis of anti-B19V NS1 IgG and $\mathrm{IgM}$, respectively.

\section{Materials and methods}

\subsection{Clinical specimens}

Three groups of sera were used in this study. (i) Specimens $(n=19)$ were obtained from children (age range: 7-11 years) within 3 months of a school-based outbreak of $\mathrm{B} 19 \mathrm{~V}$ infection, (ii) anti-B19V IgM negative sera $(n=17)$ were obtained from volunteer donors exhibiting no signs of recent $\mathrm{B} 19 \mathrm{~V}$ infection, and (iii) specimens $(n=40)$ that were positive for anti-B19V VP2 IgM. All sera were screened for anti-B19V IgG and IgM by US FDA cleared in-vitro diagnostic immunoassays (Biotrin, Dublin, Ireland).

\subsection{Construction of recombinant baculovirus encoding NS1}

B19V DNA template suitable for PCR was obtained from a B19V viremic specimen. Primers based on the B19V sequence to include sequences complementary to the $5^{\prime}$ and $3^{\prime}$ ends of the entire $2 \mathrm{~kb}$ NS1 open reading frame were selected. The 5'-primer (NSnt:GGTATGAATTCTACTTGTTAACATCC ) included an EcoRI site and the 3'-primer (NSc:CAAATTTATCATCATCACTTCTGCAGC ATTTGCC) included a Pst I site in order to facilitate the subsequent cloning of the PCR product into the baculovirus transfer vector, pBacPAK9 (Clontech, Palo Alto, CA) using standard molecular biology techniques. Spodoptera frugiperda $\left(\mathrm{Sf}_{9}\right)$ insect cells were cotransfected with recombinant transfer vector and Bsu36 I-linearised BacPAK-6 baculovirus using Bacfectin $^{\circledR}$ liposomal preparation (Clontech). Well plates were infected with the co-transfection mixtures and the TC-100 supernatant was harvested after 5 days' incubation at $27^{\circ} \mathrm{C}$. A pure clone of a recombinant baculovirus was identified by plaque assay. 


\subsection{Antigen expression and purification}

Recombinant baculovirus encoding the NS1 gene was used to infect $\mathrm{Sf} 9$ cells in monolayer culture at a multiplicity of infection of 2.1. Infected cells $\left(5 \times 10^{8}\right)$ were harvested 4 days postinfection. SDS-PAGE and Western Blot were used to confirm the presence of recombinant B19V NS1. The cells were then lysed in the presence of protease inhibitors $(0.1 \mathrm{mM}$ phenyl methyl sulfonyl fluoride, $2 \mu \mathrm{g} / \mathrm{ml}$ of pepstatin and $2 \mu \mathrm{g} / \mathrm{ml}$ of leupeptin) by the addition of phosphate-buffered saline- $0.5 \%(\mathrm{w} / \mathrm{v})$ sodium deoxycholate. After centrifugation $(3000 \times g$ for 5 $\mathrm{min}$ ), the insoluble fraction that contained recombinant NS1 and nuclei was resuspended in $25 \mathrm{mM}$ Hepes, $5 \mathrm{mM} \mathrm{MgSO}_{4}$, pH 7.4, and subjected to DNase (Sigma, Poole, UK) treatment (final concentration: $10 \mu \mathrm{g} / \mathrm{ml}$ ) for $1 \mathrm{~h}$ at room temperature. Following a brief centrifugation and wash step $(3000 \times g$ for $3 \mathrm{~min})$, the insoluble NS1 was resuspended in $0.5-1.0 \mathrm{ml}$ of $6 \mathrm{M}$ guanidinium thiocyanate at a concentration of $4-5 \mathrm{mg} / \mathrm{ml}$ and stored at $-20^{\circ} \mathrm{C}$.

\subsection{B19V NS1 IgG Western blot}

Purified NS1 was subjected to SDS-PAGE by layering $26 \mu \mathrm{g}$ of NS1 across the stacking gel in a final volume of $250 \mu \mathrm{l}$ of solubilisation buffer (Laemmili, 1970). Electrotransfer onto nitrocellulose was carried out according to standard protocols except that $10 \mathrm{mM}$ 3-[cyclohexylamino]-1-propanesulphonic acid (CAPS), $\mathrm{pH}$ 10 , containing $10 \%(\mathrm{v} / \mathrm{v})$ methanol was used to facilitate electrotransfer. After electrotransfer and membrane blocking in $5 \%(\mathrm{w} / \mathrm{v})$ milk powder $/$ phosphate-buffered saline- $-0.05 \% \quad$ (v/v) Tween-20 ${ }^{\circledR}$ (PBST), the nitrocellulose sheets were cut into $4 \mathrm{~mm}$ wide strips $(1 \mu \mathrm{g} \mathrm{NS1} /$ strip). Specimens were diluted $1 / 100$ in $1 \%(\mathrm{w} / \mathrm{v})$ milk powder/PBST, added to individual strips and incubated for $3 \mathrm{~h}$ at room temperature. After a washing step, horse-radish peroxidase (HRP)conjugated anti-human IgG (Dako, Glostrup, Denmark) was added to the membrane and incubated for $1 \mathrm{~h}$ at room temperature. Bound antibody-conjugate complex was detected by the addition of $\mathrm{H}_{2} \mathrm{O}_{2}$ and diaminobenzidine (Sigma, Poole, UK). The band intensity was determined visually, and a positive result was indicated by the appearance of a band corresponding to the position of NS1 $(77 \mathrm{kDa})$.

\subsection{B19V NS1-IgM enzyme immunoassay}

Prior to testing all specimens were adsorbed using anti-human IgG adsorbent (Incstar, Stillwater, MN) by adding $10 \mu \mathrm{l}$ of serum to $150 \mu \mathrm{l}$ of adsorbent. After a brief centrifugation step $(15000 \times g$ for $2 \mathrm{~min})$, the entire supernatant was added to $850 \mu \mathrm{l}$ of PBST (final dilution: $1 / 100)$ and mixed gently. Diluted specimens were then added to microwells that had been previously coated with recombinant NS1 at $0.7 \mu \mathrm{g} / \mathrm{ml}$ in $50 \mathrm{mM}$ sodium carbonate $\mathrm{pH}$ 9.6. Following a $1 \mathrm{~h}$ incubation at room temperature and a wash step, HRP-labelled rabbit anti-human IgM (Sigma, Poole, UK) was added for $30 \mathrm{~min}$ to detect any anti-B19V NS1 IgM present. This complex was then detected by addition of tetramethyl benzidine substrate. Sulphuric acid (1 $\mathrm{N})$ addition terminated the reaction after 10 min, followed by absorbance measurement at $450 / 630 \mathrm{~nm}$. The immunoassay cut-off value was determined by obtaining the mean absorbance \pm 2S.D. $(0.183 \pm(2(0.077))=0.338)$ of a panel of anti-B19V VP2 IgM negative specimens. Test specimens with an index value (specimen/cut-off value ratio) greater than 1.0 were deemed positive for anti-B19V NS1 IgM.

\subsection{B19V NS1 IgM Western blot}

Conditions for the detection of anti-B19V NS1 IgM were identical to those described for anti-B19V NS1 IgG detection, except that all specimens were pre-treated as described for the B19V NS1 IgM enzyme immunoassay (above) and detected using HRP-labelled rabbit anti-human IgM (Sigma, Poole, UK).

\section{Results}

A single clone termed pBac11.9 was identified that contained an insert of the appropriate size. 
Sequence analysis of $\mathrm{pBac11.9}$ from the 5'- and 3'-termini using an ABI 310 Genetic analyser (PE Applied Biosystems, Foster City, CA) confirmed that the inserted PCR fragment encodes the full-length open reading frame for B19V NS1 (data not shown). For subsequent recombinant protein work, passage two viral stock was used to infect $\mathrm{Sf}_{9}$ cells. SDS-PAGE and Western blot characterisation of infected cell lysates and purified NS1 is shown in Fig. 1. It can be seen that protein of the expected size $(77 \mathrm{kDa})$ is obtained, which is strongly immunoreactive with human IgG [anti- B19V NS1]. No reactivity was observed against uninfected insect cells, although there is evidence that limited NS1 degradation has occurred during purification, as confirmed by the presence of low $M_{\mathrm{r}}$ immunoreactive fragments. After expression, it was observed that the recombinant NS1 protein was highly insoluble and required 6 $M$ guanidinium thiocyanate and $20 \mathrm{mM}$ dithiothreitol (DTT) to facilitate solubilisation, but the protein remained soluble following dialysis or dissolution, at low $\mu \mathrm{g} / \mathrm{ml}$ concentrations, into non-denaturing buffers (e.g. $50 \mathrm{mM}$ sodium carbonate $\mathrm{pH}$ 9.4). Protein quantitation (Bio-Rad, Munich, Germany) indicated a yield of $1 \mathrm{mg}$ of B19V NS1 per $10^{7}$ infected $\mathrm{Sf}_{9}$ cells.

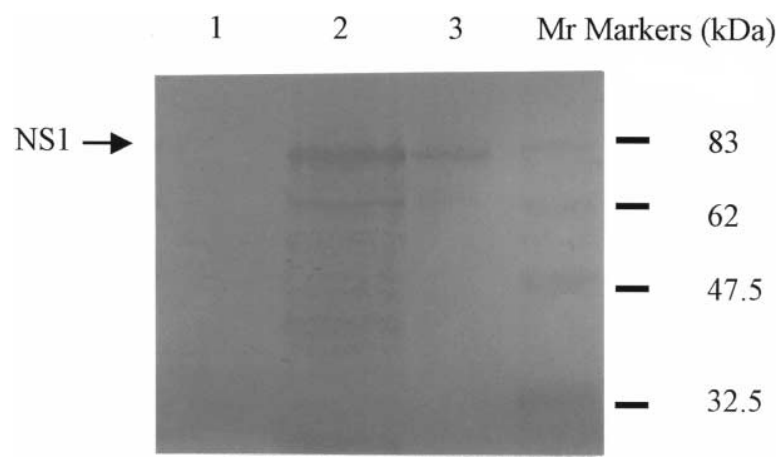

Fig. 1. Western blot analysis of baculovirus-expressed B19V NS1. No immunoreactivity is observed in uninfected insect cell lysate (1). Immunoreactivity is observed at the expected $M_{\mathrm{r}}(77$ $\mathrm{kDa}$ ) in both NS1 recombinant-baculovirus infected insect cell lysate (2) and purified NS1 (3). Antigen visualisation was carried out using an anti-B19V NS1 IgG positive specimen, as described in Table 1.
Table 1

Anti-B19V IgG and $\operatorname{IgM}$ antibody reactivity against baculovirus-expressed VP2 and NS1 antigens

\begin{tabular}{|c|c|c|}
\hline & \multicolumn{2}{|c|}{ Anti-B19V NS1 IgG status } \\
\hline & Positive & Negative \\
\hline $\begin{array}{l}\text { Anti-B19V VP2 IgG } \\
\text { positive (16) }\end{array}$ & $10(62.5 \%)$ & $6(37.5 \%)$ \\
\hline \multirow{3}{*}{$\begin{array}{l}\text { Anti-B19V VP2 IgG } \\
\text { negative (3) }\end{array}$} & $0(0 \%)$ & $3(100 \%)$ \\
\hline & \multicolumn{2}{|c|}{ Anti-B19V NS1 IgM status } \\
\hline & Positive & Negative \\
\hline $\begin{array}{l}\text { Anti-B19V VP2 IgM } \\
\text { positive (40) }\end{array}$ & $11(27.5 \%)$ & $29(72.5 \%)$ \\
\hline $\begin{array}{l}\text { Anti-B19V VP2 IgM } \\
\text { negative (17) }\end{array}$ & $1(5.9 \%)$ & $16(93 \%)$ \\
\hline
\end{tabular}

When the sera of 16 children who had been infected with B19V 3 months earlier were examined by Western blot, it was found that $11 / 16(68.8 \%)$ contained anti-B19V NS1 IgG, while $100 \%$ contained IgG against B19V VP2. No anti-B19V NS1 or VP2 IgG was detected in the sera of three seronegative children who had not been infected during the outbreak.

When tested by B19V NS1 IgM ELISA, 11/40 $(27.5 \%)$ anti-B19V VP2 IgM positive specimens also exhibited anti-B19V NS1 IgM positivity (Table 1). Subsequent Western blot analysis of 10 specimens (five anti-B19V NS1 IgM positive and five anti-B19V NS1 IgM negative by ELISA) produced a negative test result (data not shown). Only one specimen obtained from either clinical laboratories or laboratory volunteers with or without previous exposure to B19V tested anti-B19V NS1 IgM positive (Table 1). Eleven of these specimens were anti-B19V VP2 IgG positive.

\section{Discussion}

Here, we report the cloning and expression of the full-length open reading frame encoding the NS1 protein of B19V in the baculovirus expres- 
sion system. The potential diagnostic utility of the resultant purified NS1 protein has been explored, and it is clear that the antigen facilitates the detection of both anti-B19V NS1 IgG and IgM following infection with B19V. This finding offers the possibility of using anti-B19V NS1 IgM to aid confirmation of recent $\mathrm{B} 19 \mathrm{~V}$ infection in parallel with anti-B19V VP2 IgM positivity and PCR testing.

The high-level expression and purification of intact B19V NS1 protein were achieved. No evidence of NS1 cytotoxicity was observed, as has been reported for other recombinant expression systems used to produce the NS1 protein (von Poblotzki et al., 1995a,b). This is most likely due to the late protein expression obtained for any gene under the control of the baculovirus polh promoter in infected insect cells. The yield of purified NS1 was between 2 and $10 \mathrm{mg}$ per $10^{8}$ cells, which is within the expected range for the baculovirus expression system (O'Reilly et al., 1994).

Initial reports suggested that the presence of anti-B19V NS1 IgG was a strong indicator of persistent viral infection (von Poblotzki et al., 1995a,b). However, recent work in this area has indicated that there may not be a significant difference between anti-B19V NS1 IgG prevalence between individuals with either chronic or uncomplicated past B19V infection (Jones et al., 1999). Interestingly, the high level of anti-B19V NS1 IgG positivity in recently infected children observed in the present study $(68.8 \%)$ is similar to that seen for recently infected pregnant women (61\%) (Hemauer et al., 2000). Taken together, these data suggest that levels of anti-B19V NS1 IgG may peak within months after infection due to NS1 presence during viral replication and then wane as the virus is completely cleared.

Although a number of groups have undertaken the expression of $\mathrm{B} 19 \mathrm{~V} \mathrm{NS1}$ in bacterial and baculovirus expression systems, there has been no report to date on the successful detection of antiB19V NS1 IgM in specimens obtained from recently infected individuals (von Poblotzki et al., 1995a,b; Hicks et al., 1996; Venturoli, 1998; Hemauer et al., 2000). It is conceivable that the absence of relevant conformational epitopes on the NS1 antigens (or NS1 sub-fragments) previously employed resulted in the observed false negative results. Alternatively, the attempted detection of anti-B19V NS1 IgM in pooled specimens may also have lead to the conclusion that anti-B19V NS1 IgM is not present in previously infected individuals (Hicks et al., 1996). We propose that the dilution of denatured and reduced NS1 into high-pH buffer prior to microplate coating facilitates the reformation of relevant conformational epitopes that are subsequently recognised by anti-B19V NS1 IgM present in sera obtained from certain individuals recently infected with B19V. Evidence for this comes from the results obtained following Western blot analysis of anti-B19V NS1 IgM positive specimens, whereby SDS denaturation and DTT presence apparently lead to the disruption of conformationally relevant epitopes with concomitant false negative detection of anti-B19V NS1 IgM.. Since it has been previously shown that all specimens that are anti-B19V VP2 IgM positive by microplate ELISA also test positive by Western blot (Kerr et al., 1999), which used a similar method for antigen electrotransfer onto nitrocellulose, it is unlikely that methodological differences account for the observed difference with respect to antiB19V NS1 IgM detection between microplate ELISA and Western blot immunoassay. It is notable that no specimen tested was anti-B19V VP2 IgM negative and anti-B19V NS1 IgM positive, which is not unexpected given the antigenic nature of viral capsid proteins. A single specimen taken from an individual with no recent indication of B19V infection (Group (ii)) did test anti-B19V NS1 IgM positive, but since this specimen also exhibited strong $\mathrm{IgG}$ reactivity against B19V VP2, it is likely to represent a false positive reaction in the B19V NS1 IgM immunoassay.

It should now be possible to further utilise baculovirus-expressed recombinant NS1 to determine the seroprevalence of anti-B19V NS1 IgG in selected population groups, particularly in recently infected, immunocompetent individuals to confirm the high prevalence of anti-B19V NS1 $\mathrm{IgG}$ in recent infection. Significantly, Kaikkonen et al. (1999) have recently identified a specific heptapeptide epitope in B19V VP2 which is recog- 
nised by patients with acute or recent infection. Thus, it is tempting to speculate that anti-B19V NS1 IgG reactivity against specific B19V NS1 epitopes could be used, in association with antibody detection of linear epitopes on B19V VP2, as a marker of recent infection. Finally, the association between anti-B19V NS1 IgM detection and infection with specific isolates of B19V and putative associated pathogenicities, in both immunocompetent and immunocompromised individuals, also merits further investigation.

\section{Acknowledgements}

The financial assistance of Enterprise Ireland and the Irish Health Research Board is gratefully acknowledged.

\section{References}

Anderson MJ, Higgins PG, Davis LR, Willman LS, Jones SE, Kidd IM, Pattison JR, Tyrrell DAJ. Experimental parvoviral infection in humans. J Infect Dis 1985;152(2):257-65.

Brown CS, Salimans MMM, Noteborn MHM, Harro TW. Antigenic parvovirus B19 coat proteins VP1 and VP2 produced in large quantities in a baculovirus expression system. Virus Res 1990;15:197-212.

Hemauer A, Gigler A, Searle K, Beckenlehner K, Raab U, Broliden K, Wolf H, Enders G, Modrow S. Seroprevalence of parvovirus B19 NS1-specific IgG in B19-infected and uninfected individuals and infected pregnant women. J Med Virol 2000;60(1):48-55.

Hicks KE, Cubel RCN, Cohen BJ, Clewley JP. Sequence analysis of a parvovirus B19 isolate and baculovirus expression of the non-structural protein. Arch Virol 1996; 141:1319-27.

Jones LP, Erdman DD, Anderson LJ. Prevalence of antibodies to human parvovirus B19 nonstructural protein in persons with various clinical outcomes following B19 infection. J Infect Dis 1999;80(2):500-4.

Jordan JA. Identification of human parvovirus B19 infection in idiopathic nonimmune hydrops fetalis. Am J Obstet Gynecol 1996;174:37-42.

Kaikkonen L, Lankinen H, Harjunpaa I, Hokynar K, Soderlund-Venermo M, Oker-Blom C, Hedman L, Hedman K. Acute-phase-specific heptapeptide epitope for the diagnosis of parvovirus B19 infection. J Clin Micro 1999;37(12):3952-6.

Kerr S, O'Keeffe G, Kilty C, Doyle S. Undenatured parvovirus B19 antigens are necessary for the accurate serodiagnosis of parvovirus B19 IgG. J Med Virol 1999;52:179-85.

Kurtzman GJ, Ozawa K, Cohen B, Hanson G, Oseas R, Blaese RM, Young NS. Chronic bone marrow failure due to persistant B19 parvovirus infection. New Engl J Med 1987;317:287-94.

Laemmili UK. Cleavage of structural proteins during the assembly of the head of bacteriophage T4. Nature 1970;227:680-5.

Ozawa K, Young N. Characterisation of capsid and noncapsid proteins of B19 parvovirus propagated in human erythroid bone marrow. J Virol 1987;61(8):2627-30.

Török TJ. Unusual clinical manifestations reported in patients with parvovirus B19 infection. In: Anderson LJ, Young NS, editors. Human Parvovirus B19, Monographs in Virology, vol. 20. New York: Karger, 1997:61-82.

Venturoli S, Gallinella G, Manaresi E, Gentilomi G, Musiani $\mathrm{M}$, Zerbini M. IgG response to the immunoreactive region of parvovirus B19 nonstructural protein by immunoblot assay with a recombinant antigen. $\mathbf{J}$ Infect Dis 1998;178:1826-9.

von Poblotzki A, Gigler A, Lang B, Wolf B, Modrow S. Antibodies to parvovirus B19 NS1 protein in infected individuals. J Gen Virol 1995a;76:519-27.

von Poblotzki A, Hemauer A, Gigler A, Puchhammer-Stockl E, Heinz FX, Pont J, Laczika K, Wolf H, Modrow S. Antibodies to the nonstructural protein of parvovirus B19 in persistently infected patients: implications for pathogenesis. J Infect Dis 1995b;172(5):1356-9.

O'Reilly DR, Miller LK, Luckow VA. An overview of baculoviruses. In: O'Reilly DR, Miller LK, Luckow VA, editors. Baculovirus Expression Vectors — a Laboratory Manual. New York: Oxford University Press, 1994:27-9. 